\title{
Central line-associated bloodstream infections in Australian ICUs: evaluating modifiable and non-modifiable risks in Victorian healthcare facilities
}

\author{
T. SPELMAN ${ }^{1 *}$, D. V. PILCHER ${ }^{2,3,4}$, A. C. CHENG ${ }^{5}$, A. L. BULL ${ }^{1}$, \\ M. J. RICHARDS ${ }^{1,6}$ AND L. J. WORTH ${ }^{1,6}$ \\ ${ }^{1}$ Victorian Healthcare Associated Infection Surveillance System (VICNISS) Coordinating Centre, Melbourne, \\ Victoria, Australia \\ ${ }^{2}$ The Australian and New Zealand Intensive Care Society (ANZICS) Centre for Outcome and Resource \\ Evaluation, Melbourne, Victoria, Australia \\ ${ }^{3}$ Department of Epidemiology and Preventive Medicine, The Australian and New Zealand Intensive Care \\ Research Centre, Monash University, Melbourne, Victoria, Australia \\ ${ }^{4}$ Department of Intensive Care, The Alfred Hospital, Melbourne, Victoria, Australia \\ ${ }^{5}$ Department of Epidemiology and Preventive Medicine, Faculty of Medicine, Nursing and Health Sciences, \\ Monash University, Melbourne, Victoria, Australia \\ ${ }^{6}$ Department of Medicine, University of Melbourne, Melbourne, Victoria, Australia
}

Received 17 March 2017; Final revision 3 August 2017; Accepted 3 August 2017;

first published online 4 September 2017

\section{SUMMARY}

Central line-associated bloodstream infections (CLABSIs) in intensive care units (ICUs) result in poor clinical outcomes and increased costs. Although frequently regarded as preventable, infection risk may be influenced by non-modifiable factors. The objectives of this study were to evaluate organisational factors associated with CLABSI in Victorian ICUs to determine the nature and relative contribution of modifiable and non-modifiable risk factors. Data captured by the Australian and New Zealand Intensive Care Society regarding ICU-admitted patients and resources were linked to CLABSI surveillance data collated by the Victorian Healthcare Associated Infection Surveillance System between 1 January 2010 and 31 December 2013. Accepted CLABSI surveillance methods were applied and hospital/patient characteristics were classified as 'modifiable' and 'non-modifiable', enabling longitudinal Poisson regression modelling of CLABSI risk. In total, 26 ICUs were studied. Annual CLABSI rates were 1·72, 1·37, 1.00 and 0.93/1000 CVC days for 2010-2013. Of non-modifiable factors, the number of non-invasively ventilated patients standardised to total ICU bed days was found to be independently associated with infection (RR 1.07; 95\% CI 1.01-1.13; $P=0 \cdot 030$ ). Modelling of modifiable risk factors demonstrated the existence of a policy for mandatory ultrasound guidance for central venous catheter (CVC) localisation (RR $0 \cdot 51 ; 95 \%$ CI $0 \cdot 37-0 \cdot 70 ; P<0 \cdot 001)$ and increased number of sessional specialist full-time equivalents (RR $0 \cdot 52 ; 95 \%$ CI $0 \cdot 29-0 \cdot 93 ; P=0 \cdot 027$ ) to be independently associated with protection against infection. Modifiable factors associated with reduced CLABSI risk include ultrasound guidance for CVC localisation and increased availability of sessional medical specialists.

Key words: Central line-associated bloodstream infection (CLABSI), preventable, risk, ultrasoundguidance.

\footnotetext{
* Author for correspondence: T. Spelman, Victorian Healthcare Associated Infection Surveillance System (VICNISS) Coordinating Centre, Doherty Institute, 792 Elizabeth Street, Melbourne, Victoria 3000, Australia. (Email: tim@burnet.edu.au)
} 


\section{INTRODUCTION}

Central line-associated bloodstream infections (CLABSIs) in intensive care units (ICUs) result in increased morbidity, prolonged hospitalisation and greater healthcare expenditure $[1,2]$. CLABSI events are frequently regarded as preventable, leading to public reporting of infection rates [3], 'zero tolerance' in many programs [4], and inclusion in pay-for-performance healthcare funding schemes [5]. These healthcare-associated infections are therefore considered key quality indicators.

Over the last decade, the impact of multi-modal interventions (bundles of care) for reduction in CLABSI rates in ICUs has been evaluated [6, 7]. The underlying premise is that modifiable risk factors for infection can be mitigated by implementation of effective quality improvement programs. However, risks for infection may also be influenced by nonmodifiable factors within a healthcare facility (such as case-mix) [4, 8], and it has been estimated that up to $35 \%$ of CLABSI events may not be preventable $[9,10]$. This means that even with quality improvement efforts, a zero CLABSI rate may not be achievable or sustainable [11]. Few have evaluated the characteristics of healthcare facilities to determine the nature of non-modifiable factors contributing to risk of infection $[4,11,12]$.

CLABSI events in Victorian ICUs are monitored using uniform surveillance methods consistent with the Centers for Disease Control/National Healthcare Safety Network (CDC/NHSN) [13]. Annual review of ICU resources and services, including factors potentially associated with infection risk, is performed by the Australian and New Zealand Intensive Care Society (ANZICS) Centre for Outcome and Resource Evaluation (CORE) [14]. Since 2012, ANZICS CORE has also collated and reported annual ICU CLABSI rates throughout Australia and New Zealand. The objectives of this study were to use these datasets to evaluate organisational factors associated with CLABSIs in Victorian ICUs to determine the nature and relative contribution of modifiable and non-modifiable risk factors.

\section{METHODS}

The Victorian Healthcare Associated Infection Surveillance System (VICNISS) Coordinating Centre was established for the purpose of monitoring a range of healthcare-associated infection outcomes and relevant processes in Victorian hospitals, including CLABSI [15]. The CLABSI surveillance module is based upon methods employed by the $\mathrm{CDC} /$ NHSN [16, 17].

All Australian and New Zealand ICUs are invited to participate in the ANZICS CORE registry benchmarking programs and software is provided to units to assist in data collection. Patient level data on diagnosis, severity of illness and outcomes are submitted quarterly to the ANZICS Adult Patient Database. In addition, ICUs are surveyed annually to obtain information on resources (e.g. ICU bed numbers), processes of care (e.g. compliance with accredited care standards) and staffing levels (e.g. number of nursing staff), which is collected by the ANZICS Critical Care Resources Registry. These data support research on resourcing and planning, disease trends and effectiveness of quality improvement programs [14].

\section{Participating hospitals}

Victorian ICUs provide services for mixed medical and surgical patient populations rather than as singlespecialty units [18]. Hospitals participating in both the VICNISS program and ANZICS CORE activities for adult ICUs between 1 January 2010 and 31 December 2013 were included in this analysis, excluding those with paediatric and neonatal ICUs.

\section{Definitions}

For the purposes of surveillance, a central venous catheter (CVC) was defined as an intravascular catheter terminating at or close to the heart or in one of the great vessels, which was used for infusion, withdrawal of blood, or haemodynamic monitoring. Consistent with CDC/NHSN criteria, CLABSI was defined when (i) a patient had a recognised pathogen cultured from one or more blood cultures, and the organism was not related to infection at another site, or (ii) a patient had one or more of fever $\left(>38^{\circ} \mathrm{C}\right)$, chills or hypotension, a common commensal organism was cultured from two or more blood cultures drawn on separate occasions, and positive results were not related to infection at another site [17]. CLABSI rates were reported using the denominator of CVC days.

Captured hospital-level characteristics were defined as 'modifiable' and 'non-modifiable' risk factors by a consensus involving three clinicians (LW, AC and DP). Modifiable factors were regarded as reversible 
Table 1. Patient and hospital-level characteristics potentially associated with infection risk: classification of modifiable and non-modifiable factors

\begin{tabular}{ll}
\hline \hline Modifiable factors & Non-modifiable factors \\
\hline - Any infective diagnosis & - Annual number of patients with known mortality \\
- Ventilated in first $24 \mathrm{~h}$ & - Age \\
- Policy of mandatory ultrasound guidance for CVC localisation & - APACHE III score \\
- Number of registered nurse FTE & - Number of invasive ventilation \\
- Total invasively ventilated hours & \\
- Total non-invasively ventilated hours & \\
- Number of salaried specialists FTE & \\
- Number of sessional specialists FTE & \\
- Total number of non-intensive care specialists FTE & \\
\hline \hline
\end{tabular}

CVC, central venous catheter; FTE, full-time equivalent; APACHE, acute physiology and chronic health evaluation

and amenable to change according to allocated financial or human resources. Classification of hospitaland patient-level characteristics is summarised in Table 1.

\section{Data collection and validation}

Data regarding CLABSI events were collected and submitted quarterly by infection prevention staff affiliated with participating healthcare facilities. All staff were trained regarding case-definitions and standardized data collection by VICNISS Coordinating Centre staff. All data were submitted electronically via a standardised data collection tool. As an internal validation measure, electronic submission precluded lodgement of incomplete or inconsistent data. Prospective monitoring was performed, with contribution from patient records and microbiology reports.

\section{Data linkage}

Data from the VICNISS surveillance program and ANZICS datasets were linked at the level of the ICU site using a unique ICU identification number.

\section{Statistical analyses}

Categorical variables were summarised using frequency and percentage. Continuous variables were summarised using mean and standard deviation (s.D.) or median and inter-quartile range (IQR), as appropriate. Correlates of CLABSI risk across the observation period were modelled using unadjusted and adjusted longitudinal Poisson regression. CLABSI counts were tested for over-dispersion. Effect sizes were quantified as relative risk (RR) while cumulative period central line days were specified as the offset exposure variable. A goodness-of-fit $\chi^{2}$ test was used to assess overall model fit. Modelling was performed across all factor types and sub-group analyses were then performed for sets of pre-specified modifiable and non-modifiable covariates. A test of interaction was used to test for subgroup effects. For all analysis, $P<0.05$ was considered significant. All analyses were undertaken using Stata version 14 (StataCorp, College Station, Texas).

\section{Ethics}

The study was reviewed and approved by the Alfred Health Human Research Ethics Committee. No patient-identifying data were captured for the purposes of the study, and all hospital-level data were de-identified.

\section{RESULTS}

During the study period, a total of 37 public and private hospitals were resourced to provide adult ICU care in Victoria. Of these, 26 hospitals $(70 \cdot 3 \%$ of total), including 20 public and six private facilities, participated in both the VICNISS program and ANZICS CORE activities and were therefore eligible for inclusion. All studied ICUs had competency standards for CVC insertion, ward rounds with an infectious diseases specialist or microbiologist, rounds with a pharmacist, an antimicrobial stewardship program, infection prevention policy, generation of regular antibiograms specific to the ICU and a critical incident monitoring program.

The aggregate annual CLABSI rate for studied ICUs was $1 \cdot 72(95 \%$ confidence interval (CI) $1 \cdot 40-2 \cdot 08) / 1000$ CVC days in 2010, $1 \cdot 37$ (95\% CI 1.08-1.70)/1000 CVC 


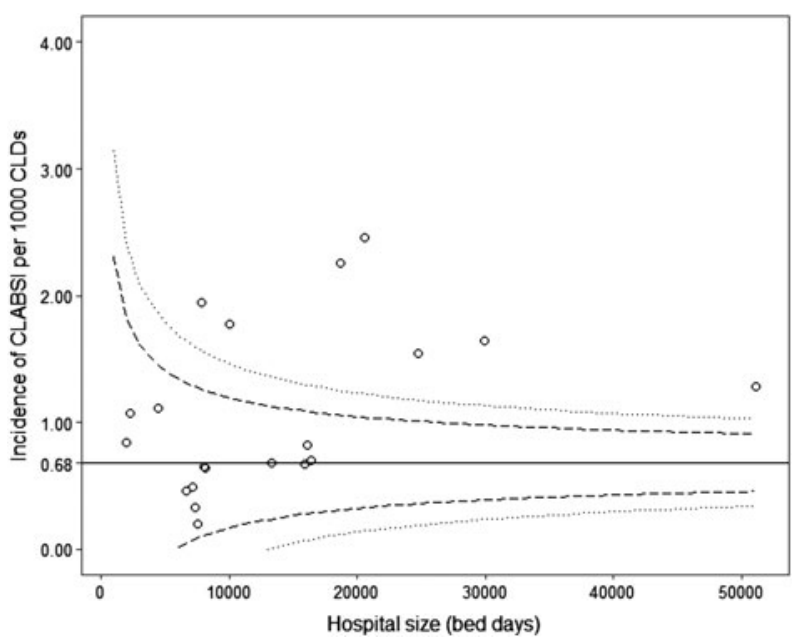

Fig. 1. Funnel plot of CLABSI rates according to hospital size (2010-2013). CLABSI, central line associated bloodstream infection; CLD, central line days.

days in 2011, $1 \cdot 00$ (95\% CI 0.75-1·30)/1000 CVC days in 2012 and $0.93(95 \%$ CI $0 \cdot 69-1 \cdot 24) / 1000$ CVC days in 2013. Mean incidence was 0.83 CLABSI per 1000 CVC days (s.D. 0.73). Six sites recorded zero CLABSI cases across the observation period. The distribution of site-level CLABSI incidence as a function of hospital size (bed days) is provided as a funnel plot in Figure 1 . The median age of ICU-admitted patients was 66 years (IQR 64.4-68.5). Characteristics of ICU-admitted patients during the study period are summarised in Table 2.

The median number of beds in studied ICUs was 12 (IQR 8-15), with median bed occupancy of $77 \%$. Allocated resources for participating ICUs included median nursing and medical full-time equivalents of 49.8 (IQR 32.5-81.7) and $1 \cdot 2(0-4 \cdot 5)$ per ICU per year, respectively. Table 3 summarises key site characteristics of participating ICUs.

In the combined model containing all available potential patient- and hospital-level risk factors for infection, independent risk factors included annual number of patients with known mortality (RR $1 \cdot 11 ; 95 \%$ CI $1 \cdot 04-1 \cdot 19 ; P=0.002)$, median APACHE III scores (RR $1.03 ; 95 \%$ CI $1.01-1.06 ; P=0.031$ ), total hours invasively ventilated (RR 1.14; 95\% CI 1.08-1.21; $P<$ $0.001)$, and total hours non-invasively ventilated (RR $1 \cdot 01 ; 95 \%$ CI $1 \cdot 01-1 \cdot 02 ; P<0 \cdot 001)$. Factors independently associated with reduced infection risk included ventilation in first $24 \mathrm{~h}$ (RR 0.85, 95\% CI 0.77-0.94; $P=0.002)$ and an existing policy of mandatory ultrasound guidance for CVC localisation (RR 0.47; $95 \%$ CI $0.35-0.64 ; P<0 \cdot 001)$. Of the 26 sites included in the analysis, $12(46 \%)$ reported mandatory US guidance policy during the observation period. Table 4 summarises univariate and multivariate analyses of covariates included in the adjusted model.

When non-modifiable risk factors only were modelled, the number of non-invasively ventilated patients per total bed days was identified as an independent risk factor for infection (RR $1.07 ; 95 \%$ CI 1.01 $1 \cdot 13 ; P=0 \cdot 030)$. Older age was independently associated with protection against infection (RR 0.94; 95\% CI $0 \cdot 90-0 \cdot 99 ; P=0 \cdot 020$ ). Table 5 summarises modelling of non-modifiable risk factors for CLABSI.

When modifiable risk factors only were modelled, any form of ventilation was associated with increased risk of infection. Total invasively ventilated hours (RR $1 \cdot 12 ; 95 \%$ CI $1 \cdot 06-1 \cdot 18 ; P<0 \cdot 001)$ and total noninvasively ventilated hours (RR $1 \cdot 01 ; 95 \%$ CI 1.01 $1.02 ; P<0.001)$ were retained as independent risk factors for infection, both standardised to total ICU bed days. The existence of a policy for mandatory ultrasound guidance for CVC localisation (RR $0.51 ; 95 \%$ CI $0 \cdot 37-0 \cdot 70 ; P<0.001)$ and number of sessional specialist FTE (RR $0.52 ; 95 \%$ CI $0.29-0.93 ; P=0.027$ ) were independently associated with protection against infection. Table 6 summarises modelling of modifiable risk factors for CLABSI. When hospital ICUs identified as outliers with respect to CLABSI rates $(n=7$, Fig. 1) were compared with non-outlier facilities, there was a non-significant trend toward outlier facilities having a smaller time period in which ultrasound policy was available $(29 \%$ vs. $33 \%$ of interval years, $P=0.712$ ) and lower specialist FTE (mean 0.96 vs. 1.07 specialist FTE per 1000 bed days, $P=0.521$ ).

\section{DISCUSSION}

Our study demonstrates a relationship between development of CLABSI in ICU patients and both staff resources and CVC insertion practices. Although the disease burden and epidemiology of CLABSI in Australian ICUs has previously been reported [13, 19], the relationship between CLABSI and ICU processes and resource allocation has not been evaluated. This is therefore the first Australian study to explore organisation-wide factors, which are associated with CLABSI risk. Given the increasing use of CLABSI as a performance indicator in healthcare facilities [20], it is essential and necessary for such factors to be evaluated, in order that modifiable factors are contained within future prevention programs.

CDC consensus guidelines for the prevention of intravascular catheter-related infections include the 
Table 2. Patient characteristics: aggregate annual data for ICU-admitted patients, 2010-2013

\begin{tabular}{lc}
\hline \hline Patient characteristics & Median (IQR) \\
\hline Age (years) & $66(64 \cdot 4-68 \cdot 5)$ \\
APACHE III score & $49(46-55)$ \\
Predicted risk of death from APACHE III scoring system & $5 \cdot 14(3 \cdot 28-7 \cdot 08)$ \\
Patients with sepsis admission diagnosis code ${ }^{\mathrm{a}}$ & $45(29-76)$ \\
Patients with any infection admission diagnosis code & $101(65-149)$ \\
Patients with any infection who meet SIRS and organ dysfunction criteria in first 24 h of ICU & $29(14-47)$ \\
Burns & $0(0-1)$ \\
Metastases, lymphoma, leukaemia & $35(17-67)$ \\
Immunosupressed by disease, therapy or AIDS & $40(7-75)$ \\
Number with invasive mechanical ventilation in first 24 h of ICU & $210(75-729)$ \\
Number of first admissions to ICU (i.e. number of patients) & $765(599-1223)$ \\
Number of patients with a survival outcome listed & $741(588-1193)$ \\
Number of patients who were readmitted to ICU & $34(15-68)$ \\
Number of patients who died in hospital & $78(44-142)$ \\
Total number of ICU admissions submitted to the ANZICS APD & $795(634-1318)$ \\
In-hospital mortality (deaths/admissions) & $0 \cdot 11(0 \cdot 07-0 \cdot 13)$ \\
\hline \hline
\end{tabular}

APACHE, acute physiology and chronic health evaluation; IQR, inter-quartile range; ICU, intensive care unit; AIDS, acquired immunodeficiency syndrome; SIRS, systemic inflammatory response syndrome; ANZICS, Australia and New Zealand Intensive Care Society; APD, adult patient database.

${ }^{\text {a }}$ Sepsis admission diagnosis codes included those for sepsis or septic shock (as defined by the ANZICS modification of the APACHE scoring system).

${ }^{\mathrm{b}}$ Infection admission diagnosis codes included those for sepsis as well as pneumonia, central nervous system infection, cellulitis and localised soft tissue infections, cholecystitis/cholangitis or gastrointestinal perforation/peritonitis (as defined by the ANZICS modification of the APACHE scoring system).

Table 3. ICU annual characteristics: $2010-2013$ ( $n=26$ sites)

\section{Site characteristics}

Modifiable factors (annual)

- Number of infective diagnosis - median (IQR)

- Patients ventilated in first $24 \mathrm{~h}$ - median (IQR)

- Policy of mandatory ultrasound guidance for CVC localisation $-n(\%)$

- Number of registered nurse FTE - median (IQR)

- Total invasively ventilated hours - median (IQR)

- Total non-invasively ventilated hours - median (IQR)

- Number of salaried specialists FTE - mean (s.D.)

- Number of sessional specialists FTE - mean (S.D.)

- Total number of non-intensive care specialists FTE - mean (s.D.)

$98(67,151)$

$142(64,697)$

$12(46 \cdot 2)$

$48(25,81)$

$13357(4479,39752)$

$931(172,5391)$

$2 \cdot 6(3 \cdot 3)$

$1 \cdot 1(1 \cdot 6)$

$0 \cdot 3(0 \cdot 7)$

$700(578,1226)$

$62 \cdot 6(4 \cdot 7)$

$54 \cdot 4(7 \cdot 8)$

$48(5,294)$

$28(0,204)$

Non-modifiable factors (annual)

- Number of patients with known mortality - median (IQR)

- Age (years) - mean (s.d.)

- APACHE III score - mean (s.D.)

- Number of invasive ventilation - median (IQR)

- Number of non-invasively ventilated patients - median (IQR)

CVC, central venous catheter, APACHE, acute physiology and chronic health evaluation; IQR, inter-quartile range; ICU, intensive care unit; FTE, full time equivalent; s.D., standard deviation.

recommendation that ultrasound guidance be used to place CVCs, if this technology is available [21]. The rationale for this recommendation is that ultrasonography has been shown to reduce the number of cannulation attempts and mechanical complications [22]. Although one observational study has demonstrated no reduction in CLABSI risk with ultrasoundguided CVC insertion [23], two pooled studies spanning 
Table 4. Unadjusted and adjusted analysis of potential risk factors for CLABSI

\begin{tabular}{|c|c|c|c|c|}
\hline Characteristic & $\begin{array}{l}\text { Unadjusted RR } \\
(95 \% \mathrm{CI})\end{array}$ & $P$-value & $\begin{array}{l}\text { Adjusted RR } \\
(95 \% \mathrm{CI})\end{array}$ & $P$-value \\
\hline Any infective diagnosis ${ }^{a}$ & $1 \cdot 34(1 \cdot 15-1 \cdot 56)$ & $<0 \cdot 001$ & $1 \cdot 14(0 \cdot 88-1 \cdot 46)$ & $0 \cdot 316$ \\
\hline Ventilated in first $24 \mathrm{~h}^{\mathrm{a}}$ & $1.06(1.03-1 \cdot 09)$ & $<0 \cdot 001$ & $0.85(0 \cdot 77-0.94)$ & $0 \cdot 002$ \\
\hline Annual number of patients with known mortality ${ }^{a}$ & $1 \cdot 05(1 \cdot 03-1 \cdot 07)$ & $<0 \cdot 001$ & $1 \cdot 11(1 \cdot 04-1 \cdot 19)$ & $0 \cdot 002$ \\
\hline Median APACHE III score & $1 \cdot 05(1 \cdot 03-1 \cdot 07)$ & $<0 \cdot 001$ & $1 \cdot 03(1 \cdot 01-1 \cdot 06)$ & $0 \cdot 031$ \\
\hline $\begin{array}{l}\text { Policy of mandatory ultrasound guidance for localisation for } \\
\text { CVC cannulation }\end{array}$ & $0 \cdot 60(0 \cdot 43-0 \cdot 78)$ & $<0.001$ & $0 \cdot 47(0 \cdot 34-0 \cdot 64)$ & $<0 \cdot 001$ \\
\hline Total invasively ventilated hours per TBD & $1 \cdot 06(1 \cdot 02-1 \cdot 10)$ & $0 \cdot 001$ & $1 \cdot 14(1 \cdot 08-1 \cdot 21)$ & $<0 \cdot 001$ \\
\hline Total non-invasively ventilated hours per TBD & $1 \cdot 00(1 \cdot 00-1 \cdot 01)$ & $0 \cdot 001$ & $1 \cdot 01(1 \cdot 01-1 \cdot 02)$ & $<0 \cdot 001$ \\
\hline
\end{tabular}

CLABSI, central line-associated bloodstream infections; ICU, intensive care unit; RR, relative risk; 95\% CI, 95\% confidence interval; FTE, full-time equivalent; APACHE, acute physiology and chronic health evaluation; TBD, total ICU bed days; CVC, central venous catheter.

${ }^{\mathrm{a}}$ Units of 100 .

Table 5. Non-modifiable risk factors for CLABSI: unadjusted and adjusted analysis

\begin{tabular}{|c|c|c|c|c|}
\hline Characteristic & $\begin{array}{l}\text { Unadjusted RR } \\
(95 \% \mathrm{CI})\end{array}$ & $P$-value & $\begin{array}{l}\text { Adjusted RR } \\
(95 \% \mathrm{CI})\end{array}$ & $P$-value \\
\hline Annual number of patients with known mortality & $1 \cdot 05(1 \cdot 03-1 \cdot 07)$ & $<0 \cdot 001$ & $1 \cdot 01(0 \cdot 98-1 \cdot 05)$ & $0 \cdot 534$ \\
\hline Median age & $0.92(0.89-0.95)$ & $<0 \cdot 001$ & $0.94(0.90-0.99)$ & $0 \cdot 020$ \\
\hline Median APACHE III score & $1 \cdot 05(1.03-1 \cdot 07)$ & $<0 \cdot 001$ & a & \\
\hline Number of invasive ventilations per $100 \mathrm{TBD}$ & $1 \cdot 02(1 \cdot 01-1 \cdot 04)$ & $0 \cdot 029$ & $1 \cdot 01(0 \cdot 98-1 \cdot 03)$ & $0 \cdot 529$ \\
\hline Number of non-invasively ventilated patients per $100 \mathrm{TBD}$ & $1 \cdot 06(1 \cdot 01-1 \cdot 12)$ & $0 \cdot 012$ & $1 \cdot 07(1 \cdot 01-1 \cdot 13)$ & $0 \cdot 030$ \\
\hline
\end{tabular}

CLABSI, central line-associated bloodstream infections; RR, relative risk; 95\% CI, 95\% confidence interval; APACHE, acute physiology and chronic health evaluation; TBD, total ICU bed days.

${ }^{\text {a }}$ Given the inclusion of age in APACHE III, this variable is not included independently in multivariate model.

a range of patient populations and medical devices have demonstrated benefit for prevention of CLABSI [24, 25]. Our findings concur with these pooled studies, and support the routine use of ultrasound guidance for CVC localisation as a means of reducing risk of CLABSI by approximately $50 \%$. Recognising that the ICU medical workforce may not be fully trained in ultrasound technique, we support current international consensus guidelines but also suggest evaluation of workforce skills be performed to determine the feasibility of routine ultrasound guidance in all Victorian centres.

Furthermore, we identified staff resources, particularly the availability of sessional medical specialists as contributing significantly to reduced CLABSI risk. This is consistent with the fact that practice in Australian ICUs is for medical staff (rather than nursing staff) to insert CVCs, and that increased medical staffing may therefore increase the available time for optimal CVC insertion practices. Others have reported limited clinical staffing as a risk for
CLABSI in neonatal ICUs [26] and nursing staff reductions as a contributing factor to increased CLABSI risk [27, 28]. In contrast, we did not identify nursing staff resources as a significant contributor to CLABSI risk. These findings may be uniquely relevant to the workforce employed in Australian ICUs. Increased sessional, but not salaried medical specialist resources, were associated with reduced risk for CLABSI. In Australia, the appointment of sessional specialists may occur in the setting of service expansion and increased funding for an ICU, and sessional staff may be visiting medical officers with appointment across multiple ICUs within a number of healthcare facilities. In addition, these staff tend to be specialists who have more recently completed final exit specialisation exams in Intensive Care practice. It is possible that some of these factors could impact upon CLABSI risk within an ICU - for example, a mobile workforce may be more obliged to be familiar with local infection prevention policies, may be more likely to be up-to-date with recent infection control 
Table 6. Modifiable risk factors for CLABSI: unadjusted and adjusted analysis

\begin{tabular}{|c|c|c|c|c|}
\hline Characteristic & $\begin{array}{l}\text { Unadjusted RR } \\
(95 \% \mathrm{CI})\end{array}$ & $P$-value & $\begin{array}{l}\text { Adjusted RR } \\
(95 \% \mathrm{CI})\end{array}$ & $P$-value \\
\hline Any infective diagnosis ${ }^{\mathrm{a}}$ & $1 \cdot 13(1 \cdot 15-1 \cdot 56)$ & $<0 \cdot 001$ & $1 \cdot 28(0 \cdot 99-1 \cdot 65)$ & $0 \cdot 061$ \\
\hline Ventilated in first $24 \mathrm{~h}^{\mathrm{a}}$ & $1.06(1.03-1.09)$ & $<0.001$ & $0.97(0.92-1.03)$ & $0 \cdot 308$ \\
\hline $\begin{array}{l}\text { Policy of mandatory ultrasound guidance for localisation for } \\
\text { central venous cannulation }\end{array}$ & $0 \cdot 60(0 \cdot 43-0 \cdot 78)$ & $<0.001$ & $0 \cdot 51(0 \cdot 37-0 \cdot 70)$ & $<0 \cdot 001$ \\
\hline Number of registered nurse FTE per 100 TBD & $0 \cdot 94(0 \cdot 82-1 \cdot 08)$ & 0.393 & $0 \cdot 86(0 \cdot 67-1 \cdot 11)$ & $0 \cdot 251$ \\
\hline Total invasively ventilated hours per TBD & $1 \cdot 06(1 \cdot 02-1 \cdot 10)$ & $0 \cdot 001$ & $1 \cdot 12(1 \cdot 06-1 \cdot 18)$ & $<0 \cdot 001$ \\
\hline Total non-invasively ventilated hours per TBD & $1.00(1 \cdot 00-1 \cdot 01)$ & $0 \cdot 001$ & $1.01(1.01-1 \cdot 02)$ & $<0 \cdot 001$ \\
\hline Number of salaried specialists FTE per 1000 TBD & $1 \cdot 01(0 \cdot 79-1 \cdot 30)$ & 0.963 & $0 \cdot 81(0 \cdot 52-1 \cdot 24)$ & $0 \cdot 330$ \\
\hline Number of sessional specialists FTE per 1000 TBD & $0 \cdot 66(0 \cdot 48-0 \cdot 91)$ & $0 \cdot 012$ & $0 \cdot 52(0 \cdot 29-0 \cdot 93)$ & 0.027 \\
\hline $\begin{array}{l}\text { Total number of non-intensive care specialists FTE per } 1000 \\
\text { TBD }\end{array}$ & $0.67(0.50-0.90)$ & 0.007 & $0 \cdot 36(0 \cdot 06-2 \cdot 27)$ & $0 \cdot 278$ \\
\hline
\end{tabular}

CLABSI, central line-associated bloodstream infections; RR, relative risk; 95\% CI, 95\% confidence interval; FTE, full-time equivalent; TBD, total ICU bed days.

${ }^{\mathrm{a}}$ Units of 100.

literature and more likely to use ultrasound routinely for CVC insertion.

We also demonstrated that any form of ventilation was associated with increased risk of CLABSI. While the reasons for this are not clear, others have also observed an association between infection and mechanical ventilation [29, 30], and it is possible that respiratory infection in the setting of ventilation could predispose to bloodstream infection, or that patients in the ICU environment may become colonised at multiple sites (e.g. respiratory tract and CVC) with pathogens such as MRSA and Candida spp., both of which are recognised causes of CLABSI [31].

When hospital ICUs identified as outliers in the current study with respect to CLABSI rates (Fig. 1) were compared with non-outlier facilities, we observed a trend toward smaller time period in which ultrasound policy was available and lower specialist FTE. Although not statistically significant, this observation is consistent with risk factor analysis performed in our study and the fact that these modifiable factors hold clinical significance.

The context for this study must be understood prior to applying findings to populations in other regions. A national hand-hygiene program has been operational in Australia for 7 years, and compliance with WHO moments for hand hygiene is a focus for high risk wards, including ICU staff [32]. Also, all ICUs included in our study had competency standards for CVC insertion, ward rounds with an infectious diseases specialist or microbiologist, rounds with a pharmacist, an antimicrobial stewardship program, infection prevention policy, generation of regular antibiograms specific to the ICU and a critical incident monitoring program. Regionally, quality improvement has been a focus in all Victorian hospitals, with infections in ICUs comprising one element of statewide performance monitoring [33]. This is reflected by the observation that overall CLABSI rates diminished during the studied 4 year period (from $1 \cdot 72$ to $0 \cdot 93 / 1000 \mathrm{CVC}$ days).

We identified older age as being protective for CLABSI, and this contrasts with other studies reporting increased risk for CLABSI in elderly patients [34]. While the reasons for this are not clear, it is possible that the studied population represented a cohort of elderly patients with higher functional capacity and reduced infection risk, as these patients had been admitted to ICU and were considered eligible for active care. Conversely, those patients with poorer prognosis (and potentially higher risk for CLABSI) would be more likely to be managed conservatively in a ward environment, and therefore not have been included in the current study. In a large retrospective study of ICU-admitted patients spanning 15 years, Blot et al. reported decreased incidence of bloodstream infections in elderly ICU patients, with the proportion of all bloodstream infections in very elderly patients attributed to a venous catheter being only $13 \%$ [35]. Results of the current study support these findings.

One limitation of our study is the fact that an existing dataset of ICU resources was used, and this has not been specifically formulated for the purposes of infection risk surveillance. Nonetheless, we believe 
many plausible risk factors for CLABSI to have been adequately captured by the dataset (e.g. age, patient co-morbidities, presence of other infections). In the absence of an accepted tool or previously published framework for classifying risk factors, the definition of modifiable and non-modifiable risk factors used in our study was based on clinical consensus. For example, the duration of ventilation for intubated patients is generally minimised by clinicians, and this was therefore regarded as a modifiable factor. The availability of non-invasive ventilatory support in a step-down or ward setting could impact the number of invasively-ventilated patients in a given $\mathrm{ICU}$, and this capacity requires dedicated resources outside of ICU. Management of concurrent infections can be optimised by early identification and targeted therapy. Prevention of concurrent healthcareassociated infections (e.g. healthcare-associated pneumonia or bloodstream infection) requires effective and appropriately resourced hospital-wide infection prevention programs (e.g. implementation of evidence-based bundles of care), therefore other infective diagnoses were similarly considered as modifiable. We believe our classification to be a viable and pragmatic basis for evaluating organisational factors amenable to human resourcing or financial support. Other limitations include the fact that studied healthcare facilities may not be representative of all Australian hospitals, potentially limiting the generalizability of findings. In addition, information from ANZICS CORE annual surveys was used, but some of this hospital-level information may have changed over time.

Study strengths include the representativeness of participating ICUs in our region, with 26/37 sites being eligible for inclusion $(70 \cdot 3 \%$ ), all of which were evaluated in the current study. Also, we believe that partitioning of modifiable and non-modifiable risks is a pragmatic and necessary step to adequately inform prevention strategies at the hospital-level. Quality improvement programs can then focus upon those factors that are potentially reversible, in order to optimise outcomes and rationalise resources.

\section{ACKNOWLEDGEMENTS}

The authors thank the infection prevention consultants in participating Victorian hospitals for collecting and submitting data regarding CLABSI events. ICU staff are acknowledged for submission of ANZICS data. The Victorian Healthcare Associated Infection
Surveillance System is fully funded by the Victorian Department of Health and Human Services.

\section{REFERENCES}

1. Zimlichman E, et al. Health care-associated infections: a meta-analysis of costs and financial impact on the US health care system. JAMA Internal Medicine 2013; 173: 2039-2046.

2. Leistner R, et al. Costs and prolonged length of stay of central venous catheter-associated bloodstream infections (CVC BSI): a matched prospective cohort study. Infection 2014; 42: 31-36.

3. Marsteller JA, Hsu YJ, Weeks K. Evaluating the impact of mandatory public reporting on participation and performance in a program to reduce central line-associated bloodstream infections: evidence from a national patient safety collaborative. American Journal of Infection Control 2014; 42(10 Suppl): S209-2115.

4. Worth LJ, McLaws ML. Is it possible to achieve a target of zero central line associated bloodstream infections? Current Opinion in Infectious Diseases 2012; 25: 650-657.

5. Centers for Medicare and Medicaid Services (CMS), HHS. Medicare program; hospital inpatient prospective payment systems for acute care hospitals and the longterm care hospital prospective payment system and fiscal year 2013 rates; hospitals' resident caps for graduate medical education payment purposes; quality reporting requirements for specific providers and for ambulatory surgical centers. Final rule. Federal Register 2012; 77: 53257-53750.

6. Tang HJ, et al. The impact of central line insertion bundle on central line-associated bloodstream infection. BMC Infectious Diseases 2014; 14: 356.

7. Klintworth G, et al. Beyond the intensive care unit bundle: implementation of a successful hospital-wide initiative to reduce central line-associated bloodstream infections. American Journal of Infection Control 2014; 42: 685-687.

8. Fraser TG, Gordon SM. CLABSI rates in immunocompromised patients: a valuable patient centered outcome? Clinical Infectious Diseases 2011; 52: 1446-1450.

9. Septimus EJ, Moody J. Prevention of device-related healthcare-associated infections. F1000Research 2016; 5: F1000 Faculty Rev-65.

10. Umscheid CA, et al. Estimating the proportion of healthcare-associated infections that are reasonably preventable and the related mortality and costs. Infection Control and Hospital Epidemiology 2011; 32: 101-114.

11. O'Grady NP. Zero risk for central line-associated bloodstream infections ... Is this realistic? Critical Care Medicine 2012; 40: 657-658.

12. Editorial. Set a goal of zero central line and VAP infections. Determining what is really preventable. Hospital Peer Review 2008; 33: 4-5.

13. Worth LJ, et al. Central line-associated bloodstream infections in Australian intensive care units: time-trends in infection rates, etiology, and antimicrobial resistance 
using a comprehensive Victorian surveillance program, 2009-2013. American Journal of Infection Control 2015; 43: 848-852.

14. Sanagou M, et al. Associations of hospital characteristics with nosocomial pneumonia after cardiac surgery can impact on standardized infection rates. Epidemiology and Infection 2016; 144: 1065-1074.

15. Russo PL, et al. The establishment of a statewide surveillance program for hospital-acquired infections in large Victorian public hospitals: a report from the VICNISS coordinating centre. American Journal of Infection Control 2006; 34: 430-436.

16. Centers for Diseases Control and Prevention. The National Healthcare Safety Network (NHSN) Patient Safety Component Manual. 2017 (http://www.cdc.gov/ nhsn/PDFs/pscManual/PSC-Manual-portfolio.pdf).

Last accessed February 2017.

17. Edwards JR, et al. National Healthcare Safety Network (NHSN) report: data summary for 2006 through 2008, issued December 2009. American Journal of Infection Control 2009; 37: 783-805.

18. ANZICS Centre for Outcome and Resource Evaluation Annual Report 2012-2013, ANZICS Melbourne. 2014 (http://www.anzics.com.au/Downloads/ANZICS\%20 CORE\%20Annual\%20Report\%202013.pdf). Last accessed February 2017.

19. Wong SW, et al. The influence of intensive care unit-acquired central line-associated bloodstream infection on in-hospital mortality: a single-center risk-adjusted analysis. American Journal of Infection Control 2016; 44: 587-592.

20. Barnes H, Rearden J, McHugh MD. Magnet ${ }^{\circledR}$ hospital recognition linked to lower central line-associated bloodstream infection rates. Research in Nursing and Health 2016; 39: 96-104.

21. O'Grady NP, et al. Guidelines for the prevention of intravascular catheter-related infections. Clinical Infectious Diseases 2011; 52: e162-e193.

22. Froehlich CD, et al. Ultrasound-guided central venous catheter placement decreases complications and decreases placement attempts compared with the landmark technique in patients in a pediatric intensive care unit. Critical Care Medicine 2009; 37: 10901096.

23. Cartier V, et al. No association between ultrasoundguided insertion of central venous catheters and bloodstream infection: a prospective observational study. Journal of Hospital Infection 2014; 87: 103-108.

24. Randolph AG, et al. Ultrasound guidance for placement of central venous catheters: a meta-analysis of the literature. Critical Care Medicine 1996; 24: 2053-2058.

25. Hind D, et al. Ultrasonic locating devices for central venous cannulation: meta-analysis. British Medical Journal 2003; 327: 361.

26. Leistner R, et al. The impact of staffing on central venous catheter-associated bloodstream infections in preterm neonates - results of nation-wide cohort study in Germany. Antimicrobial Resistance and Infection Control 2013; 2: 11.

27. Fridkin SK, et al. The role of understaffing in central venous catheter-associated bloodstream infections. Infection Control and Hospital Epidemiology 1996; 17: 150-158.

28. Hugonnet S, Chevrolet JC, Pittet D. The effect of workload on infection risk in critically ill patients. Critical Care Medicine 2007; 35: 76-81.

29. Pawar M, et al. Central venous catheter-related blood stream infections: incidence, risk factors, outcome, and associated pathogens. Journal of Cardiothoracic and Vascular Anesthesia 2004; 18: 304-308.

30. Al-Rawajfah OM, Stetzer F, Hewitt JB. Incidence of and risk factors for nosocomial bloodstream infections in adults in the United States, 2003. Infection Control and Hospital Epidemiology 2009; 30: 1036-1044.

31. Wang FD, et al. Risk factors and mortality of nosocomial infections of methicillin-resistant staphylococcus aureus in an intensive care unit. Journal of Critical Care, 2011; 26: 82-88.

32. Grayson ML, et al. Outcomes from the first 2 years of the Australian National Hand Hygiene Initiative. Medical Journal of Australia 2011; 195: 615-619.

33. Department of Health. Victorian Health Service Performance Monitoring Framework 2013-14 Business Rules [Internet]. Melbourne (AUST): State Government of Victoria. 2014 (http://www.health.vic.gov.au/hospitalperformance). Last accessed February 2017.

34. Yahav D, et al. Bloodstream infections in older patients. Virulence 2016; 7: 341-352.

35. Blot S, et al. Epidemiology and outcome of nosocomial bloodstream infection in elderly critically ill patients: a comparison between middle-aged, old, and very old patients. Critical Care Medicine 2009; 37: 1634-1641. 